\title{
Proton Acceleration by Very Impulsive Flare on June 3, 2012
}

\author{
K. Kamiya, ${ }^{1)}$ K. Koga, ${ }^{1)}$ S. Masuda, ${ }^{2)}$ H. Matsumoto, ${ }^{1)}$ Y. Muraki, ${ }^{2)}$ T. Obara, ${ }^{3)}$ O. \\ Okudaira, ${ }^{4)}$ Y. Tanaka, ${ }^{5)}$ S. Shibata ${ }^{6}{ }^{6}$ and T. Goka ${ }^{1)}$
}

1) Tsukuba Space Center, JAXA, Tsukuba 305-8505, Japan

2) Institute for Space-Environment Research, Nagoya University, Nagoya 464-8601, Japan

3) Planetary Plasma and Atmosphere Research Center, Tohoku University, Sendai 980-8578, Japan

4) Planetary Exploration Research Center, Chiba Institute of Technology, Tsudanuma 275-0016, Japan

5) Department of Physics, Hiroshima University, Hiroshima 739-8526, Japan

6) Engineering Science Laboratory, Chubu University, Kasugai 487-0027, Japan

E-mail:_Kamiya.kohki@jaxa.jp, muraki@isee.nagoya-u.ac.jp

\section{abstract}

On June 3, 2012, a very impulsive solar flare was observed by the GOES, RHESSI and FERMILAT satellites. The intensity of the flare was M3.3. The hard X-ray intensity $(>100 \mathrm{keV})$ rose $\geq$ 1000 times within one minute and then decayed immediately to the background level (in less than one minute). It was an extremely impulsive flare. By chance, the SEDA-FIB solar neutron detector onboard the International Space Station detected a 5.1 $\sigma$ enhancement due to solar neutrons. Therefore, it provided a good opportunity to investigate the mechanism of instantaneous proton acceleration to a few $\mathrm{GeV}$. Based on the results of a new Monte Carlo calculation, we will show a reasonable proton acceleration model for the June 3, 2012 event. We also reexamined another very impulsive flare observed on June 10, 2010.

\section{Introduction: why we selected the June 3, 2012 event}

It is well known that high-energy particles are produced in association with solar flares. Those particles are called high-energy solar particles (SEP) or solar cosmic rays (SCR). According to local tradition, we call such particles SCR. The Sun is a typical star in our universe and situated at the nearest distance from Earth. Therefore, there is a possibility of investigating the acceleration site from Earth with the use of telescopes. And new knowledge obtained about SCR could help us to better understand the acceleration mechanism of galactic cosmic rays (GCR).

SCR entails the involvement of protons, neutrons, electrons and gamma rays. The energy spectra of these particles provide us with important information for understanding the acceleration process of SCR. ${ }^{[1]}$ For example, an important element to understanding the acceleration process is how high-energy particles are involved in these particles. On the other hand, observation using photon- and light-based telescopes will provide indispensable knowledge relative to understanding the acceleration process of SCR. ${ }^{[2]}$ The X-ray satellite GOES, UV telescope SDO/AIA, soft gamma-ray detectors RHESSI and FERMI-GBM, and X-ray telescope YOKOH are typical examples of such instruments, which are used to constantly monitor the solar surface

35th International Cosmic Ray Conference - ICRC2017

10-20 July, 2017

Bexco, Busan, Korea

Presented by K. Kamiya 
and ocassionally provide us with important information. Whenever the Sun enters the field of view, the LAT detector onboard the FERMI satellite provides invaluable data on high-energy gamma rays.

The acceleration mechanism may be understood by comparing the data on SCR with the Xray data. However, in the combination process, a problem stands in the way. That problem arises from the time lag on the particles of protons, neutrons and electrons. The charged particles are transported to Earth while trapped in the interplanetary magnetic field, and thus used to arrive on Earth later than light. The arrival time of neutrons here is delayed due to the mass of neutron. Therefore, unless we otherwise measure the energy of neutrons, the time profile could not be compared with that of light.

Here we show one of the examples. Figure 1 shows the FERMI-GBM data observed in the M7.4 flare on September 25, 2011. ${ }^{[3]}$ As you may understand from this figure, the continuous emission of intensive X-rays is recognized and it continued for 20 minutes, from 04:35 to 05:05 UT. When we consider the acceleration process of protons, we must survey three possibilities: (1) protons are presumably emitted during the 20 minutes according to the time profile of the intensity of hard X-rays; and (2) protons are accelerated gradually within 20 minutes and then fully emitted into interplanetary space at 04:53 UT. This occurs because when the momenta of protons exceed a certain value, the protons are not involved in the magnetic cavity of the acceleration site, and thus escape from the region. The other possibility that we must take into account is that (3) protons are emitted instantaneously at the maximum time of hard X-rays, say at 04:45 UT, by the presumably strong induced DC electric field. ${ }^{[4]}$

In order to discriminate these possibilities, the event observed on June 3, 2012 is one of the best candidates as both solar neutrons and high-energy gamma rays were observed. The time profile of the X-rays was observed to have a delta-function shape. It shone for only one minute and intensified by 1,000 times in the hard X-ray region $(100-300 \mathrm{keV}) \cdot{ }^{[5]}$ We thus tried to reduce the energy spectrum of solar neutrons in this flare, and then compared it with the gamma-ray data observed by the FERMI-LAT satellite. The spectrum is also compared with the recent results of a MC calculation based on GEANT 4.

Another event of the same type was observed on June 12, 2010. The FERMI-LAT team had already reported this event, which did not include high-energy neutrons. ${ }^{[6]}$ Thus, we reexamined this event here and compared its data with the results of a new Monte Carlo calculation on the production of gamma rays and solar neutrons. These results have also been reported.

This paper is organized as follows: Section 2 introduces the June 3, 2012 event, Section 3 presents the reexamination results regarding the June 12, 2010 event, and Section 4 summarizes the analysis results.
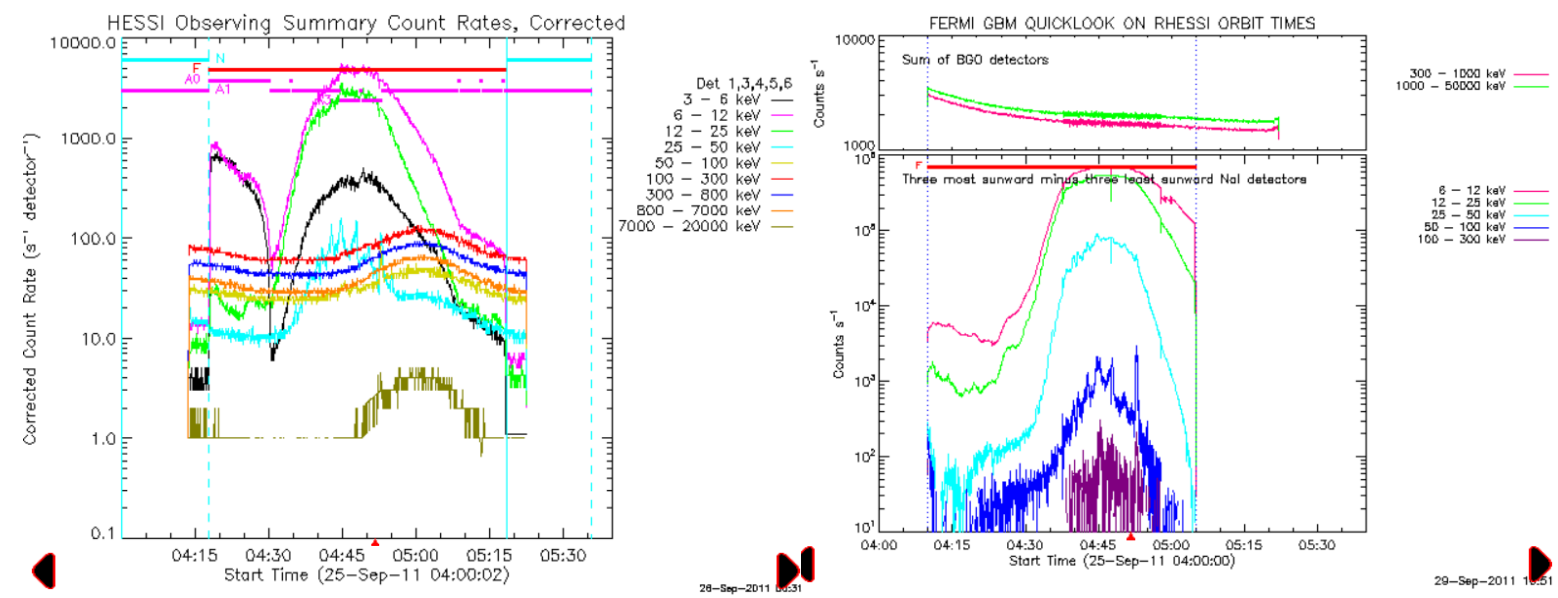

Figure 1. (left) The time profile of RHESSI and (right) of FERMI-GBM. 


\section{The June 3, 2012 event}

On June 3, 2012, a highly impulsive solar flare was observed on the solar surface. The intensity and position of the flare was M3.3 at N16E33. The soft X-ray intensities observed by the Geostationary Operational Environmental Satellite reached a maximum at 17:55 UT. Impulsive emission of hard X-rays was observed by both the Reuven Ramaty High-Energy Solar Spectroscopic Imager (RHESSI) and the Fermi Gamma-ray Burst Monitor (FERMI-GBM) satellites. They recorded very sharp rise and rapid fall-off features. Figures $\mathbf{2}$ and $\mathbf{3}$ show the data from the RHESSI and FERMI-GBM satellites, respectively. And in association with this flare, high-energy gamma rays were detected by the Fermi Large Area Telescope (FERMI-LAT) satellite. The statistical significance was $8.4 \sigma$, and the intensity was $0.77\left(\mathrm{gamma} / \mathrm{m}^{2} \cdot \mathrm{s}\right.$ at $>100$ $\mathrm{MeV}$ ). The rise time of the hard X-rays to the peak was less than 1 min., and the decay time was a few minutes. Thus, a challenging topic would be understanding how the particles are accelerated to high energies by the impulsive flares. From this viewpoint, we have analyzed the time profiles of hard X-rays, neutrons, and data obtained from the UV telescope.
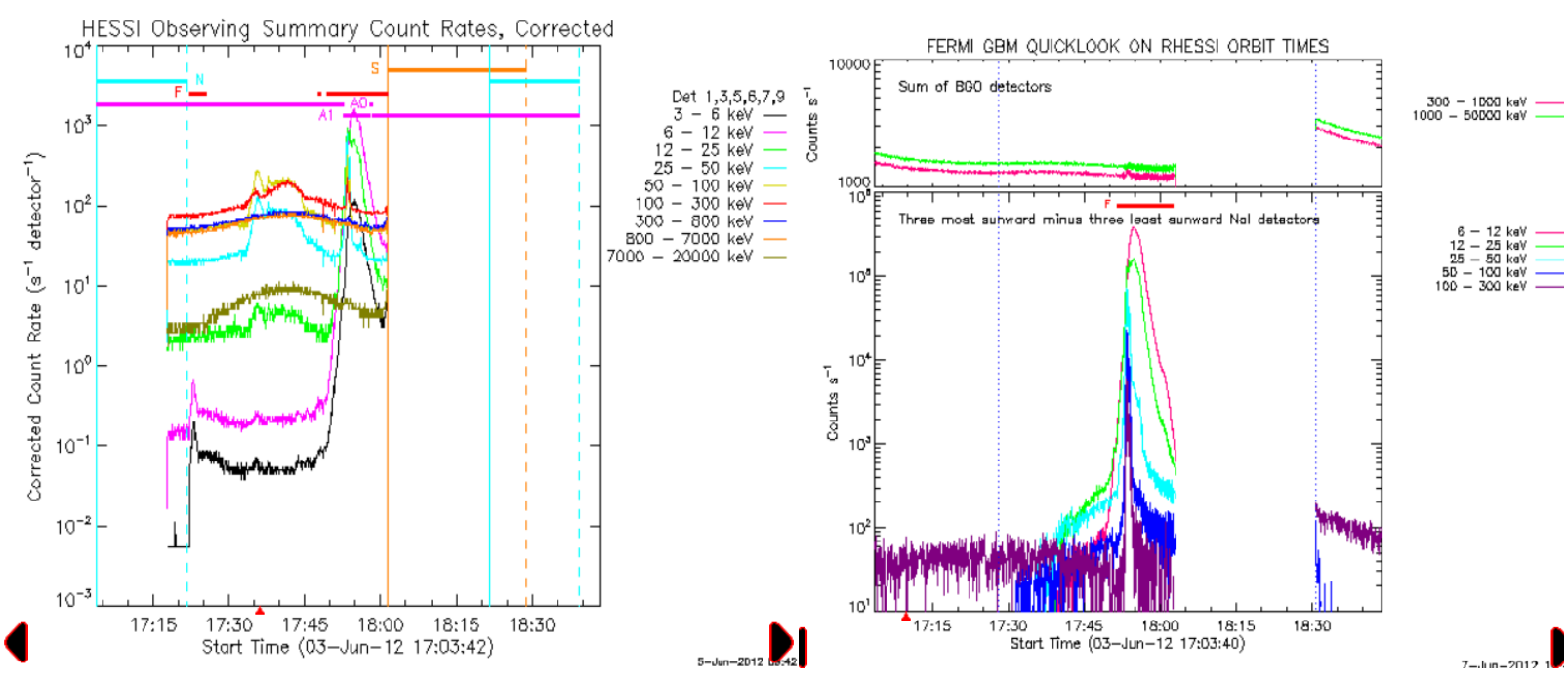

Figure 2. The time profile of RHESSI. The black curve indicates $300-800 \mathrm{keV}$ X-rays.

Figure 3. The time profile of FERMI-GBM. The purple color indicates $100-300 \mathrm{keV}$ X-rays.

We have searched for solar neutrons in the data obtained by Space Environment Data Acquisition using the FIBer detector (SEDA-FIB) onboard the International Space Station (ISS). From 17:50 to 18:30 UT, the ISS flew over the South Pacific Ocean, passing over South America's Cape Horn and the South Atlantic before reaching a point over central Africa. Between 18:13 and 18:20 UT, the ISS flew over the South Atlantic anomaly region, and we avoided analyzing data from this period. The ISS flew in daylight between 17:50 and 18:23 UT. The position of the Sun was on the opposite side of the pressurized module so as to facilitate good separation between solar and background neutrons.

Solar neutrons were identified as follows: if the track of a neutron-converted proton fell within a cone with an opening angle of less than $45^{\circ}$ from the solar direction, we identified it as being of solar origin. During the flare time, the direction of the Sun was on the opposite side of the pressurized vessel where the cosmonauts were working. Therefore, the solar neutrons and background neutrons were quite easily separated. Furthermore, we used the knowledge that lowenergy protons stop at the scintillation bar, leaving a quantity of energy and forming a Bragg peak. The deposited energy was measured by the ADC of the SEDA-FIB. To use these functions, we separated solar neutrons from background neutrons. 
We found 59 candidate solar neutrons between 17:54:24 UT and 18:13:48 UT. Figures 4 and 5 show the energy spectra determined from the arrival time in two separate cases that we assumed for the production time. One of the assumptions was that all neutrons were simultaneously produced as the soft X-rays, that is, at 17:47 UT (Fig. 4) and 17:53:15 UT (Fig. 5). The distribution at 17:47 UT shows the normal production spectrum of solar neutrons; however, we encounter a serious issue here in that at 17:47 UT, the hard X-ray intensity profile did not start high intensity emission as shown in Figs. 2 and 3. Therefore, we assumed the production time of solar neutrons to be near the peak of the hard X-rays or slightly before at 17:53:30 UT, say at 17:53:15 UT. This assumption arises from the time profile of high-energy gamma rays onboard the FERMI-LAT satellite. Figures 6 and 7 show the time profile and the energy spectrum, respectively. The origin of horizontal axis in Fig. 6 corresponds to 17:53:15 UT, and the peak time was 17:53:35 UT. The energy spectrum shows no high energy photons beyond $500 \mathrm{MeV}$. The energy spectrum of gamma rays can be fit by the power law with a power index of -3.0. In the reduction process of the energy spectrum, we have corrected the detection efficiency of gamma rays between 100 $\mathrm{MeV}$ and $600 \mathrm{MeV}{ }^{[7]}$, and also the decay effect during the flight time for neutrons.

Figure 8 shows the differential energy spectrum of solar neutrons after the correction of decay in flight. The background for neutrons is estimated as follows: we selected the data from $90 \mathrm{~min}$. before and $90 \mathrm{~min}$. after the flare time (17:50-18:30 UT), and searched for neutrons that came from the solar direction. For each of the datasets between 16:20 and 16:50 UT, and between 19:20 and 19:43 UT, we found 14 neutrons and 10 neutrons, respectively, for which the incident direction coincided with the solar direction. In this case, we avoided data analysis from the time spent passing over the SAA. In concluding this section, Figure 9 shows two SDO/AIA images.
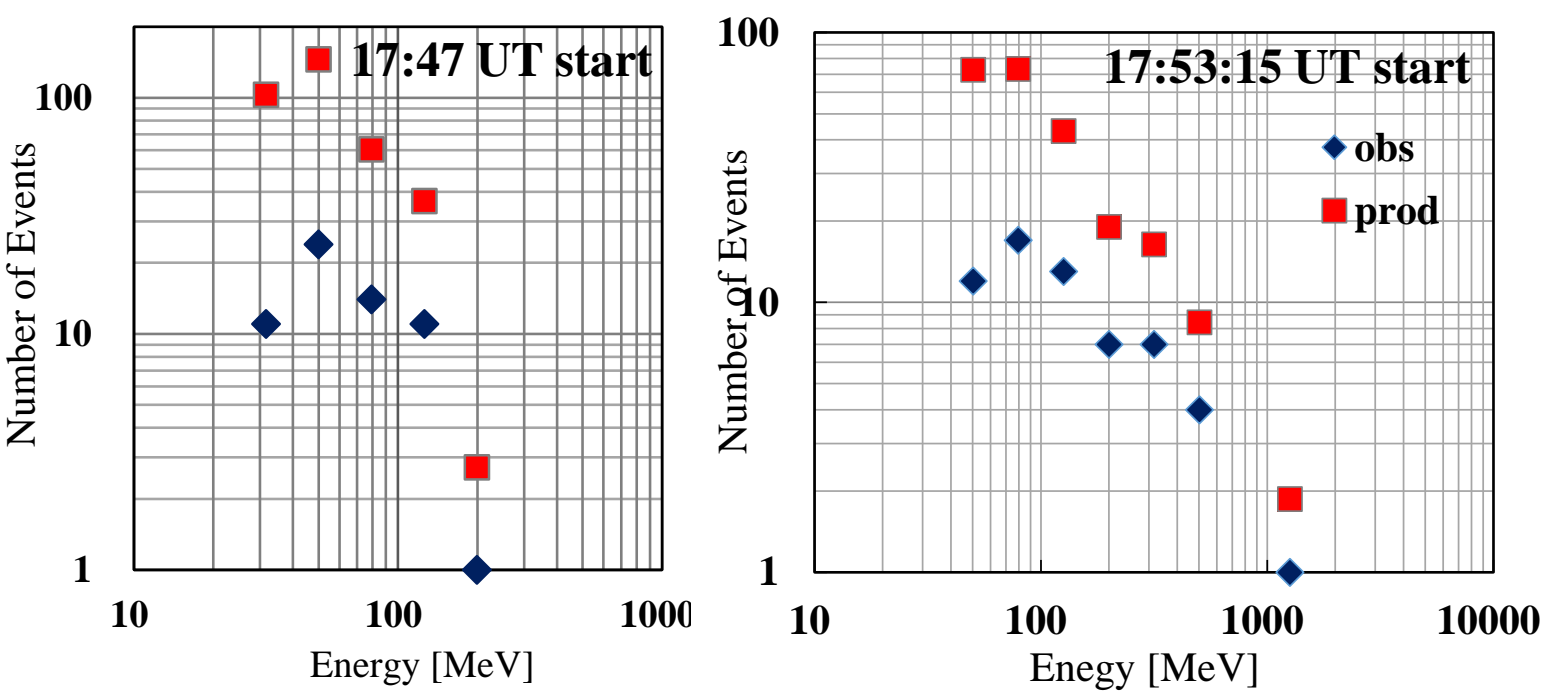

Figure 4. (Left) The energy spectrum of solar neutrons, assuming instantaneous production at 17:47 UT. Blue diamond-shape points indicate actual data; red square points correspond to the flux after correction of the decay effect in flight between the Sun and Earth.

Figure 5. (Right) The energy spectrum of solar neutrons. The energy of each neutron was determined by the flight time, assuming instantaneous production 20 seconds before the peak time of FERMI-LAT gamma rays with energy of $>100 \mathrm{MeV}$ at 15:53:15 UT. 
FERMI-LAT gamma rays

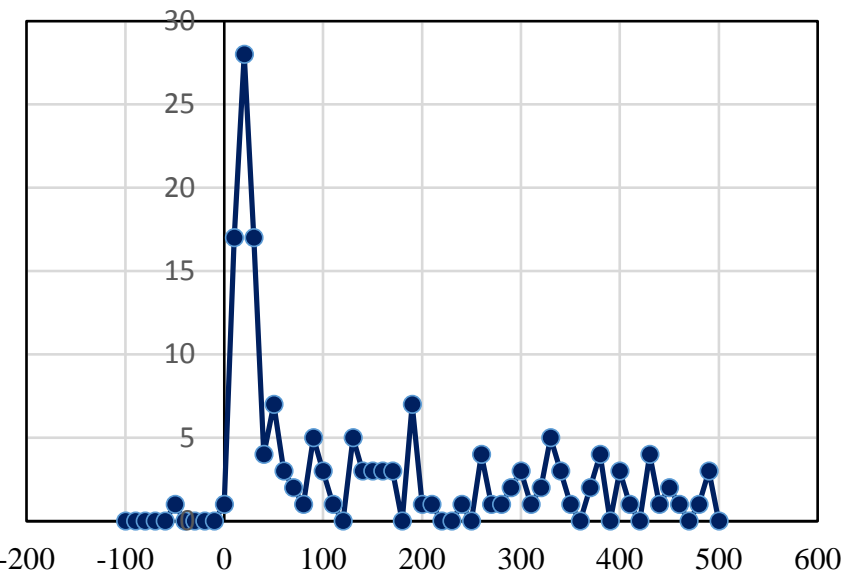

Figure 6. The time profile of high-energy gamma rays observed by the FERM-LAT. The origin of the horizontal axis is set at 17:53:15 UT and the 10 -second value is plotted. After very impulsive production of gamma rays, weak emission of high-energy gamma rays was observed for $\geq 8$ minutes.

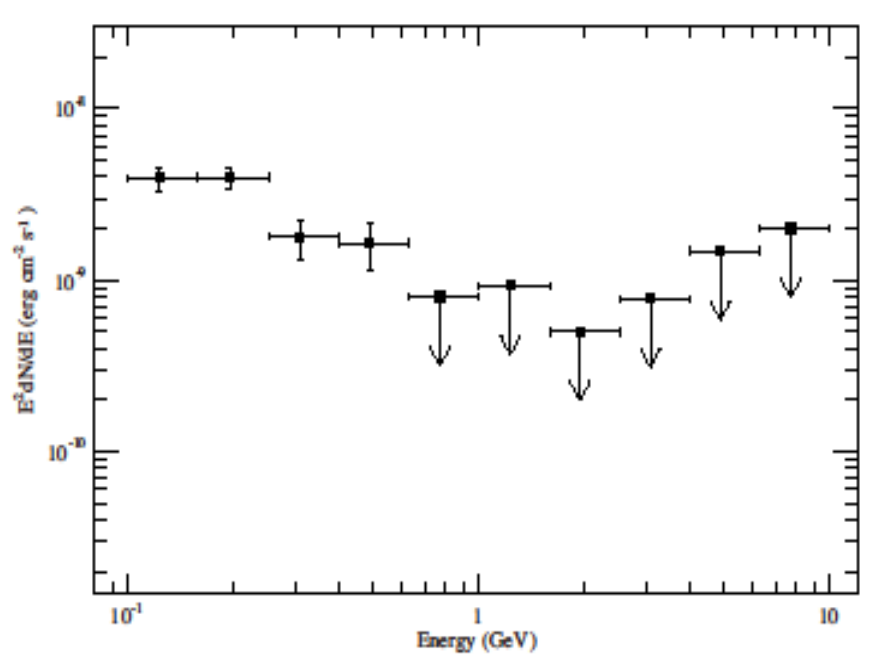

Differential energy spectrum

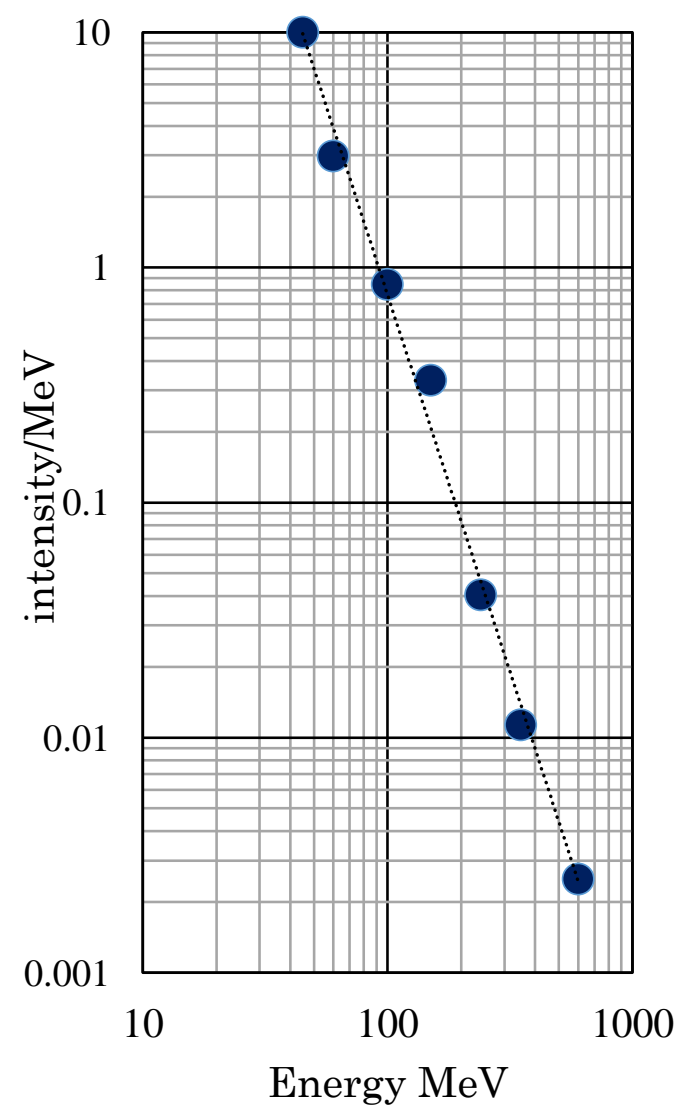

Figure 8. The differential energy spectrum of solar neutrons observed on June 3, 2012 by the SEDA-FIB detector onboard the ISS. The spectrum index may be fit to a power law with $\gamma=-3.2$.

Figure 7. The energy spectrum of high-energy gamma rays. No high-energy photon is detected beyond $600 \mathrm{MeV}$. The vertical axis is presented by the scale of $\mathrm{E}^{2} \mathrm{dN} / \mathrm{dE}$ in units of $\left[\mathrm{erg} / \mathrm{cm}^{2} \mathrm{sec}\right]$. By taking the detection efficiency of gamma rays at low energies into account, the energy spectrum should be corrected by the energy dependency of the detection efficiency factor $\left(1 / \sqrt{ } E_{\gamma}\right)$. The spectrum can then be fit by a power law of $\mathrm{E}_{\gamma}^{-3} \mathrm{dE}_{\gamma}$. 


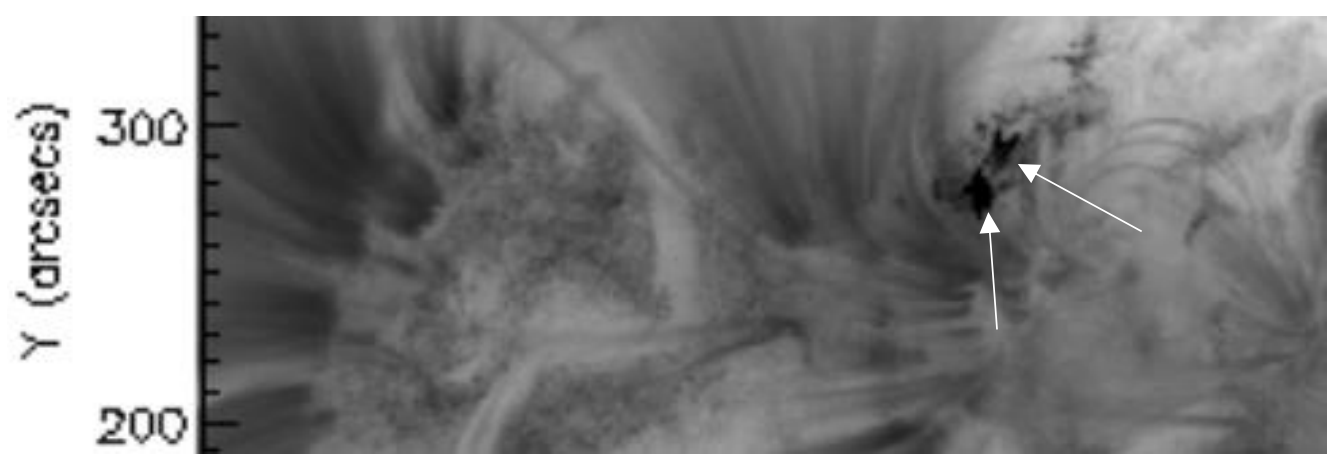

Figure 9. Images taken by the UV telescope onboard the Solar Dynamics Observatory. The image above was taken at 17:52:00 UT by the 171-nm telescope; the image below was taken at 17:52:45 UT by the 131-nm telescope. The X-type crossing of two active magnetic loops (indicated by white arrows) may be recognized.

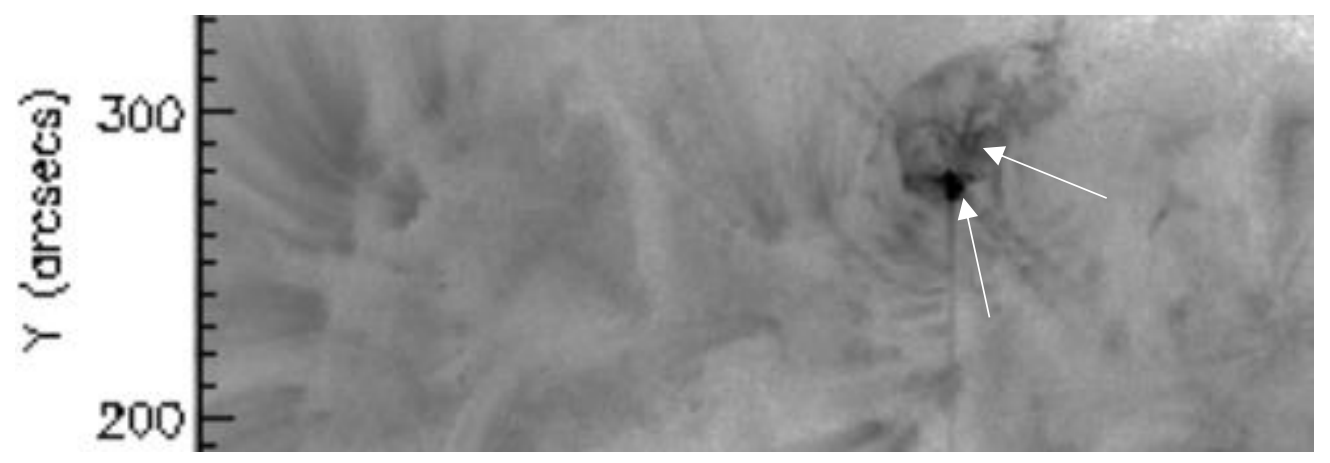

\section{The June 12, 2010 event}

Now we will discuss a similar event observed on June 12, 2012. The flare position was N23W43 and the GOES X-rays class was M2.0. Figure 10 plots the time profiles of the X-ray detectors onboard the RHESSI satellite (left) and the FERMI satellite (right). The X-ray detector FERMI-GBM in the range of 100-300 keV shows a very impulsive feature. The peak time was at 00:55 UT. We searched for solar neutrons in the data of the SEDA-FIB detector, and subsequently found 24 candidates of events coming from the solar direction.
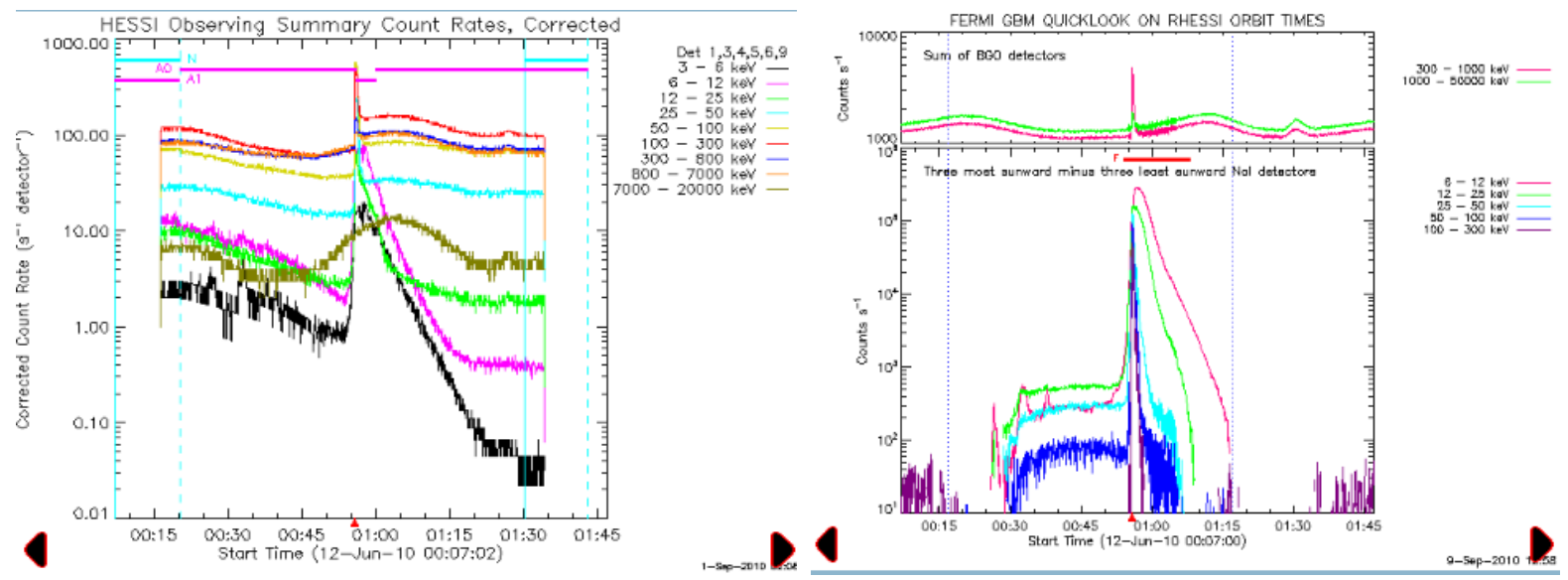

Figure 10. The time profile of the RHESSI detector (left) and the FERMI-GBM detector (right). 
Figure 11 shows the energy spectrum of solar neutrons. We assumed that those neutrons were released from the solar surface at 00:55 UT (the peak time). Conversely, the flux of gamma rays beyond $100 \mathrm{MeV}$ used the value published by the FERMI team. ${ }^{[6]}$ Here we have estimated the flux per unit square meter (although the Fermi team provided it as per square $\mathrm{cm}$ ) as follows: The number of photons between 00:55:40 and 00:56:20 UT turns out to be 235 photons $/ \mathrm{m}^{2}$. At that time, the effective area of the FERMI-LAT detector was equivalent to $0.31 \mathrm{~m}^{2}$. When taking the detection efficiency of $\sim 0.5$ for gamma rays in the low energy range into account, the net emitted intensity of gamma rays beyond $100 \mathrm{MeV}$ may be estimated to be $1,500 \mathrm{\gamma s} / \mathrm{m}^{2} / 40$ seconds. Let us compare this value with the flux of solar neutrons. In other words, neutrons are assumed to be produced during 40 seconds at the solar surface.

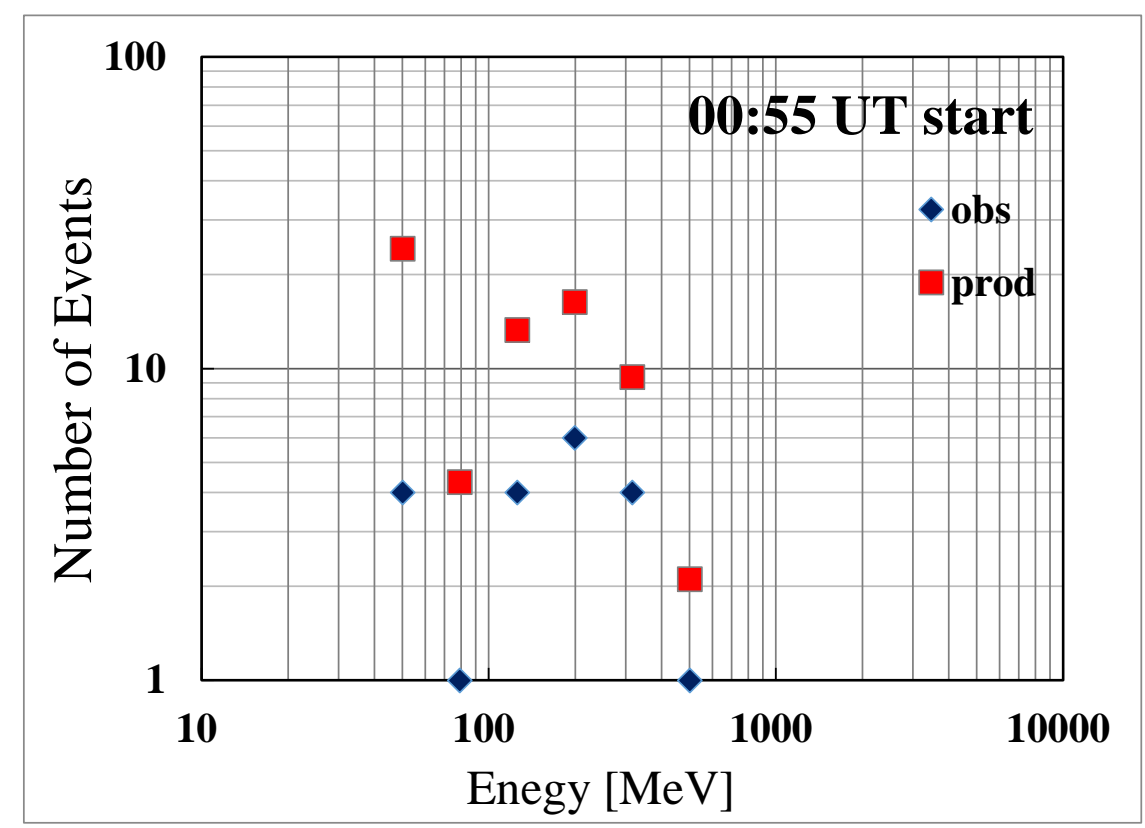

Figure 11. The energy flux of solar neutrons observed on June 12, 2010. The production time is assumed at 00:55 UT - the peak time of the hard X-rays. Red squares indicate the corrected values of the decay effect; blue diamond-shape points indicate raw data.

The detection area of SEDA-FIB is $100 \mathrm{~cm}^{2}$ and we converted it into units of $\mathrm{m}^{2}$. Then the corrected flux of neutrons are 4,200 events $/ \mathrm{m}^{2}$ for $E_{n}>100 \mathrm{MeV}$ or 7,000 events $/ \mathrm{m}^{2}$ for $E_{n}>50$ $\mathrm{MeV}$. For those values, we must further correct the chamber's detection efficiency $(\sim 0.02 \%)$. Net intensity may then be estimated at $210,000 \mathrm{n} / \mathrm{m}^{2}\left(E_{n}>100 \mathrm{MeV}\right)$ or $350,000 \mathrm{n} / \mathrm{m}^{2}\left(E_{n}>50\right.$ $\mathrm{MeV})$. This allows us to then compare the two fluxes between the gamma rays and neutrons. The value of $\mathrm{n} / \gamma$ turns out to be about $\sim 140$ beyond $>100 \mathrm{MeV}$ and $\sim 230$ beyond $>50 \mathrm{MeV}$ for the event of June 6, 2010. The abundance of neutrons could also be interpreted as forward production of the secondaries by the accelerated particles in the solar atmosphere. The accelerated protons and helium ions may be trapped in the solar magnetic field and penetrate the solar atmosphere with a large pitch angle. Looking from the Earth side, secondary neutrons and gamma rays are emitted in the forward direction $(\theta=0 \sim 40$ degrees). We have comfirmed this hypothesis by performing a Monte Carlo calculation ${ }^{[8]}$.

\section{Discussion and Summary}

Let us reduce the $\mathrm{n} / \gamma$ ratio for the event of June 3, 2012. According to Fig. 6 , the raw number of events of gamma rays between 17:53:25 and 17:55:05 UT (100 seconds) is estimated as 88 
events. For this value, we divide the detection efficiency of 0.5 for low-energy gamma rays of LAT and the effective area of $0.33 \mathrm{~m}^{2}$. Then we can obtain the number of gamma rays produced per $\mathrm{m}^{2}$ at $1 \mathrm{AU}$. The value turns out to be 533 events $/ \mathrm{m}^{2}$ during 100 seconds.

The intensity was about three times weaker than that of the event on June 12, 2010. The number of neutrons is estimated to be 75 events for $E_{n}>100 \mathrm{MeV}$ and 245 events for $E_{n}>$ $50 \mathrm{MeV}$. Again, we must convert the value into units of $\mathrm{m}^{2}$ after dividing it by the detection efficiency of $\sim 0.02$. Then the value turns out to be $375,000 \mathrm{n} / \mathrm{m}^{2}\left(\mathrm{E}_{\mathrm{n}}>100 \mathrm{MeV}\right)$ and $1.25 \times 10^{6}$ $\mathrm{n} / \mathrm{m}^{2}\left(\mathrm{E}_{\mathrm{n}}>50 \mathrm{MeV}\right)$. Compared with the numbers of the June 6, 2010 event, the neutron intensity was 9 times $\left(E_{n}>50 \mathrm{MeV}\right)$ and 18 times $\left(E_{n}>100 \mathrm{MeV}\right)$ higher in the flare of the June 3, 2012 event. And the $n / \gamma$ ratio is estimated to be $\sim 700$ in the energy region of either neutrons or gamma rays over $E_{n}$ or $E \gamma>100 \mathrm{MeV}$. Can we explain these values reasonably?

In order to understand these numbers, we examined three possibilities of the opening angle, flare point, and Earth. The two vectors are defined as the opening angle between the direction of accelerated protons and the vector defined as the direction toward Earth. The opening angles of the two flare sites of June 12, 2010 and June 3, 2012 between the vertical direction and Earth are $133^{\circ}$ and $138^{\circ}$, respectively. When the opening angle between the direction of protons and Earth is less than 40 degrees (the forward direction), the $n / \gamma$ ratio is expected to be larger than 10 ; conversly when the opening angle is larger than 90 degrees (the backward direction), the ratio becomes inverse ${ }^{[8]}$. Therefore, we believe that collisions between accelerated protons and the solar atmosphere may be produced by protons and helium ions with a large pitch angle. In the low energy helium incidence with $\mathrm{E}_{\mathrm{He}}=500 \mathrm{MeV} /$ nucleon, the $\mathrm{n} / \gamma_{\mathrm{ratio}}$ at the forward region is predicted as to be $\sim 700^{[8]}$.

\section{Acknowledgement}

The members of JAXA wish to acknowledge the efforts of staff at the Tsukuba operation centre of the Kibo operation centre in collecting the SEDA-NEM-FIB data every day.

\section{References}

[1] Miroshnichenko, Leonty (2015) Solar Cosmic Rays, $2^{\text {nd }}$ edition. ISBN 978-3-319-09428-1, published by Springer, DOI 10.1007/978-3-319-09429-8.

[2] Dorman Lev (2010) Solar Neutrons and Related Phenomena. ISBN 978-90-481-3736-7, published by Springer, DOI 10.1007/978-90-481-3737-4.

[3] http://sprg.ssl.berkeley.edu/ tohban/browser/?show=grth+qlpcr

[4] Litvinenko, Yuri (1999) Astronomy \& Astrophysics. 349 685, Also in proceedings of High Energy Solar Physics-anticipating HESSI, ASP Proceeding 206 (1999), 167, edited by Reuven Ramaty and Natalie Mandzhavidze.

[5] http://sprg.ssl.berkeley.edu/ tohban/browser/?show=grth+qlpcr

[6] Ackermann, M. et al. (2012) ApJ 745 144. Doi:10.1088/0004-637X/745/2/144.

[7] Atwood, W. B. et al. (2009) ApJ 697, (2009), 1071.

[8] Kamiya, K. et al. Proceedings of this conference paper; number \#116. 